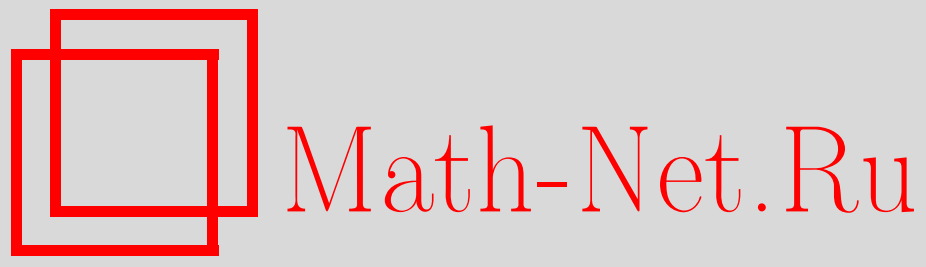

А. А. Досиев, Когомологии пучков алгебр Фреше и спектральная теория, Функи. анализ и его прил., 2005, том 39, выпуск 3, 76-80

DOI: https://doi.org/10.4213/faa76

Использование Общероссийского математического портала Math-Net.Ru подразумевает, что вы прочитали и согласны с пользовательским соглашением

http://www.mathnet.ru/rus/agreement

Параметры загрузки:

IP : 54.197 .130 .99

26 апреля 2023 г., 12:02:56

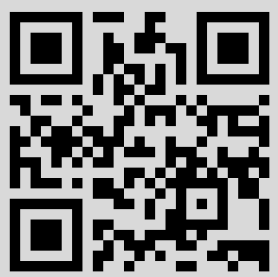




\title{
Когомологии пучков алгебр Фреше и спектральная теория
}

\author{
(c) 2005. А. А. Досиев
}

Данная заметка посвящена мультиоператорному функциональному исчислению относительно пучка некоммутативных алгебр Фреше. Предложенный ниже подход обобщает конструкцию Тейлора-Хелемского-Путинара $[1 ; 2$, гл. 6 ; $3, \mathrm{ch} .5]$, созданную для построения голоморфного исчисления набора коммутирующих операторов. Опираясь на технику когомологий пучков, методами топологической гомологии мы доказываем теорему о функциональном исчислении в окрестности спектра Путинара $\sigma(\mathscr{S}, X)$ модуля Фреше $X$ над алгеброй (Фреше) $A=\mathscr{S}(\Omega)$ глобальных сечений заданного пучка алгебр Фреше $\mathscr{S}$. При этом предполагается, что базовое пространство $\Omega$, над которым рассматривается пучок $\mathscr{S}$, удовлетворяет естественным требованием типа ацикличности по отношению к этому пучку. В качестве приложений этой теоремы мы строим пучок $\mathfrak{T}_{\mathfrak{g}}$ ростков сечений формально радикальных функций от элементов конечномерной нильпотентной алгебры Ли $\mathfrak{g}$ и доказываем теорему о функциональном исчислении для операторных семейств, порождающих конечномерную сверхнильпотентную подалгебру Ли в алгебре $\mathscr{L}(X)$ непрерывных линейных операторов, действующих в банаховом пространстве $X$. Последний результат обобщает теорему Тейлора [1] о голоморфном исчислении для коммутативных операторных семейств в $\mathscr{L}(X)$.

Всюду далее основной категорией рассматриваемых линейных пространств является категория всех комплексных пространств Фреше. Если $A$ - ассоциативная алгебра Фреше, то $A$-mod (соответственно $\bmod -A$ ) есть категория всех левых (соответственно правых) унитальных $A$-модулей Фреше. Точную проективную (соответственно свободную) резольвенту $\varepsilon: \mathscr{P} \rightarrow X$ модуля $X \in A$-mod назовем проективной (соответственно свободной) Tоr-резольвентой, если все пространства $\operatorname{Tor}_{n}^{A}(Y, X), n \in \mathbb{Z}_{+}[2$, п. 3.4.4], построенные с помощью допустимых проективных резольвент, являются гомологиями комплекса $Y \widehat{\otimes}_{A} \mathscr{P}: 0 \leftarrow$ $Y \widehat{\otimes}_{A} P_{0} \stackrel{1 \otimes_{A} d_{0}}{\longleftarrow} Y \widehat{\otimes}_{A} P_{1} \stackrel{1 \otimes_{A} d_{1}}{\longleftarrow} \ldots$ для любого $Y \in \bmod -A$. Например, если $A=$ $\mathscr{O}(\Omega)$ - алгебра Фреше всех голоморфных функций на штейновой области $\Omega$ в $\mathbb{C}^{m}$, то комплекс Кошуля $A$-бимодуля $\mathscr{O}(\Omega \times \Omega)$ является свободной точной резольвентой бимодуля $\mathscr{O}(\Omega)$ длины $m[1$, Proposition 4.3]. Отметим, что он является допустимой резольвентой, если $\Omega-$ полиобласть. Таким образом, если $X \in A$-mod, то комплекс Кошуля модуля $X$ является свободной Tor-резольвентой длины $m$.

Пусть теперь $X \in A$-mod и $Y \in \bmod -A$. Мы пишем $Y \perp_{A} X$, если $\operatorname{Tor}_{n}^{A}(Y, X)$ $=\{0\}, n \in \mathbb{Z}_{+}$. Далее, мы скажем, что модуль $X \in A$-mod является конечносвободным, если он имеет свободную Тог-резольвенту конечной длины. Из сказанного выше следует, что всякий модуль над алгеброй $\mathscr{O}(\Omega)$, где $\Omega$ - штейнова область, конечно-свободен.

1. Пучки Фреше. Пусть $\Omega$ - хаусдорфово топологическое пространство и $\mathscr{S}$ - пучок пространств Фреше (или, короче, пучок Фреше) с базой $\Omega$. Когомологии пространства $\Omega$ со значениями в пучке $\mathscr{S}[4$, п. 2.4.4] обозначаются через $H^{n}(\Omega, \mathscr{S}), n \in \mathbb{Z}_{+}$. Пусть $U \subseteq \Omega$ - открытое подмножество и $\mathscr{S} \mid U-$ пучок с 
базой $U$, индуцированный пучком $\mathscr{S}$. Говорят, что $U$ является $\mathscr{S}$-аиикличным, если $H^{n}(U, \mathscr{S} \mid U)=\{0\}, n \in \mathbb{N}$. Наконец, будем говорить, что пучок $\mathscr{S}$ с базой $\Omega$ является ядерным пучком, если $\mathscr{S}(U)$ является ядерным пространством для любого открытого $\mathscr{S}$-ацикличного подмножества $U \subseteq \Omega$.

Если $E-$ пространство Фреше, то пучок ростков голоморфных $E$-значных функций над пространством $\Omega=\mathbb{C}^{m}$ обозначается через $\mathscr{O} \widehat{\otimes} E$. Пусть $\mathscr{C}_{0, k}^{\infty} \widehat{\otimes} E$ - пучок Фреше ростков $E$-значных внешних $C^{\infty}$-дифференциальных форм бистепени $(0, k)$ с базой $\mathbb{C}^{m}$. Тогда все пучки $\mathscr{C}_{0, k}^{\infty} \widehat{\otimes} E, 0 \leqslant k \leqslant m$, как модули над пучком ростков $C^{\infty}$-функций $\mathscr{C}_{0,0}^{\infty}$ являются мягкими и $\bar{\partial}$-последовательность пучков

$$
0 \rightarrow \mathscr{O} \widehat{\otimes} E \longrightarrow \mathscr{C}_{0,0}^{\infty} \widehat{\otimes} E \stackrel{\bar{\partial}}{\longrightarrow} \mathscr{C}_{0,1}^{\infty} \widehat{\otimes} E \stackrel{\bar{\partial}}{\longrightarrow} \cdots \stackrel{\bar{\partial}}{\longrightarrow} \mathscr{C}_{0, m}^{\infty} \widehat{\otimes} E \rightarrow 0
$$

точна, т. е. мы имеем мягкую резольвенту пучка $\mathscr{O} \widehat{\otimes} E$. С помощью этой резольвенты доказывается (см. [5, п. 4.2.6; 3, Appendix 1]), что открытое множество $U \subseteq \mathbb{C}^{m}$ является $\mathscr{O} \widehat{\otimes} E$-ацикличным, если $U$ псевдовыпукло.

Пусть $\mathscr{S}$ - пучок Фреше с базой $\Omega$. Мы говорим, что $\Omega$ является локалъно $\mathscr{S}$-ацикличным пространством, если каждая точка в $\Omega$ имеет фундаментальную систему открытых $\mathscr{S}$-ацикличных окрестностей.

ОПреДЕЛЕНИЕ А. Пусть $\Omega$ - локально $\mathscr{S}$-ацикличное пространство, где $\mathscr{S}$ - пучок алгебр, $A=\mathscr{S}(\Omega)$ и $X \in A$-mod. Резолъвентным множеством $\operatorname{res}(\mathscr{S}, X)$ модуля $X$ относительно пучка $\mathscr{S}$ назовем множество тех точек $\lambda \in \Omega$, для которых существует открытая окрестность $D_{\lambda}$, такая, что $\mathscr{S}(W) \perp_{A} X$ для каждого открытого $\mathscr{S}$-ацикличного подмножества $W \subseteq D_{\lambda}$. Cneктром Путинара модуля $X$ относительно пучка $\mathscr{S}$ назовем множество $\sigma(\mathscr{S}, X)=$ $\Omega \backslash \operatorname{res}(\mathscr{S}, X)$.

Из определения А непосредственно следует, что $\operatorname{res}(\mathscr{S}, X)$ открыто в $\Omega$; поэтому $\sigma(\mathscr{S}, X)$ замкнут.

ОПРЕДЕЛЕНИЕ В. Пусть $\mathscr{S}$ - пучок Фреше с базой $\Omega$. Мы говорим, что пространство $\Omega$ является $\mathscr{S}$-пространством, если оно имеет счетную базу $\mathfrak{B}$, состоящую из открытых $\mathscr{S}$-ацикличных подмножеств, таких, что $U \cap U^{\prime} \in \mathfrak{B}$ всякий раз, когда $U, U^{\prime} \in \mathfrak{B}$.

Например, комплексное пространство $\mathbb{C}^{m}$ является $\mathscr{O} \widehat{\otimes} E$-пространством. Кроме того, само пространство $\mathbb{C}^{m}$ является $\mathscr{O} \widehat{\otimes} E$-ацикличным.

Отметим, что всякое $\mathscr{S}$-пространство является локально $\mathscr{S}$-ацикличным.

ТЕОрема 1. Пусть $\Omega$ является $\mathscr{S}$-пространством, где $\mathscr{S}-$ ядерный пучок алгебр Фреше, пусть $X \in \mathscr{S}(\Omega)-\bmod -$ конечно-свободный модуль, и пусть $U$ - открытая окрестность спектра Путинара $\sigma(\mathscr{S}, X)$ в $\Omega$. Если $\Omega$ является $\mathscr{S}$-аиикличным, то $X \in \mathscr{S}(U)$-mod и его $\mathscr{S}(\Omega)$-модульная структура, индуцированная гомоморфизмом сужения $\mathscr{S}(\Omega) \rightarrow \mathscr{S}(U)$, сводится $\kappa$ изначалъной.

Рассмотрим пример, когда $\Omega$ есть штейнова область в $\mathbb{C}^{m}, \mathscr{S}=\mathscr{O}$ и $X \in$ $\mathscr{O}(\Omega)$-mod. Тогда $\Omega$ является $\mathscr{O}$-пространством. Более того, как было отмечено, $X$ является конечно-свободным $\mathscr{O}(\Omega)$-модулем. По теореме 1 мы заключаем, что $X$ превращается в левый $\mathscr{O}(U)$-модуль, структура которого совместима с $\mathscr{O}(\Omega)$-модульной структурой всякий раз, когда $U$ открыто и $\sigma(\mathscr{S}, X) \subset U($ см. [3, Theorem 5.1.5]). 
2. Формально радикальные функции. Всюду далее $\mathfrak{g}$ обозначает конечномерную нильпотентную алгебру Ли, которая допускает положительную градуировку, и пусть $e=\left(e_{1}, \ldots, e_{n}\right)$ - базис в $\mathfrak{g}$, подчиненный этой градуировке. Тогда $e_{m+1}, \ldots, e_{n}$ оказывается базисом коммутанта $[\mathfrak{g}, \mathfrak{g}]$ для некоторого $m$. Пусть $\Delta(\mathfrak{g})$ - пространство всех лиевых характеров алгебры Ли g. Ясно, что $\Delta(\mathfrak{g})$ отождествляется с комплексным пространством $\mathbb{C}^{m}$. В работах $[6,7]$, были введены алгебры Фреше-Аренса-Майкла $\mathscr{O}_{e}\left(D_{0, r}\right)$ голоморфных функций от элементов базиса $e$ на открытых полидисках $D_{0, r} \subseteq \Delta(\mathfrak{g})$ с центрами в нуле и полирадиусов $r \in \mathbb{R}_{+}^{m}$. Алгебра $\mathscr{O}_{e}\left(D_{0, r}\right)$ является пополнением (Фреше) универсальной обертывающей алгебры $\mathscr{U}(\mathfrak{g})$ алгебры Ли $\mathfrak{g}$ по некоторой системе мультипликативных преднорм, построенной по $D_{0, r}$. Отметим, что $\mathscr{O}_{e}\left(\mathbb{C}^{m}\right)$ сводится к оболочке Аренса-Майкла (см. [8, п. 5.2.21]) $\mathscr{O}_{\mathfrak{g}}$ алгебры $\mathscr{U}(\mathfrak{g})$. Пусть $a \in \Delta(\mathfrak{g}), a_{i}=a\left(e_{i}\right)$ и $\mathfrak{g}-a=\{u-a(u): u \in \mathfrak{g}\}$ - подалгебра Ли в $\mathscr{U}(\mathfrak{g})$. Алгебра голоморфных функций $\mathscr{O}_{e}\left(D_{a, r}\right)$ от базиса $e$ на открытом полидиске $D_{a, r}$ с центром в $a$ и полирадиуса $r$ определяется (см. [9]) как $\mathscr{O}_{e-a}\left(D_{0, r}\right)$. Множество $\mathfrak{m}_{e-a}$ всех упорядоченных мономов $(e-a)^{J}=\left(e_{1}-a_{1}\right)^{j_{1}} \cdots\left(e_{n}-a_{n}\right)^{j_{n}}$, $J \in \mathbb{Z}_{+}^{n}$, является однородным базисом в алгебре $\mathscr{O}_{e}\left(D_{a, r}\right)[7]$. Мы определим пространство $\mathscr{F}_{e}\left(D_{a, r}\right)$ всех формально радикалъных функиий от элементов алгебры Ли $\mathfrak{g}$ на полидиске $D_{a, r}$ как формальное пополнение алгебры $\mathscr{O}_{e}\left(D_{a, r}\right)$ по отношению к однородному базису $\mathfrak{m}_{e-a}$.

ПРЕДЛОЖЕНИЕ 2. Пространство $\mathscr{F}_{e}\left(D_{a, r}\right)$ превращается в алгебру Фреше с нильпотентной сверткой в качестве умножения, и каноническое вложение $\mathscr{O}_{e}\left(D_{a, r}\right) \rightarrow \mathscr{F}_{e}\left(D_{a, r}\right)$ является непрерывным мономорфизмом алгебр. Кроме того, всякий модуль $X \in \mathscr{F}_{e}\left(D_{a, r}\right)$-mod конечно-свободен.

3. Пучок $\boldsymbol{T}_{\mathfrak{g}}$. Пусть $E=\mathbb{C}\left[\left[\omega_{1}, \ldots, \omega_{n-m}\right]\right]-$ пространство (Фреше) всех формальных степенных рядов от $n-m$ переменных $\omega_{1}, \ldots, \omega_{n-m}$. Определим пучок ростков формально радикальных функций $\mathfrak{T}_{\mathfrak{g}}$ с базой $\Delta(\mathfrak{g})$ как пучок $\mathscr{O} \widehat{\otimes} E$ (см. п. 1). Ясно, что $\mathfrak{T}_{\mathfrak{g}}\left(D_{a, r}\right)=\mathscr{F}_{e}\left(D_{a, r}\right)$, в частности, $\mathscr{F}_{\mathfrak{g}}=\mathfrak{T}_{\mathfrak{g}}(\Delta(\mathfrak{g}))$. Более того, операцию нильпотентной свертки в алгебрах $\mathscr{F}_{e}\left(D_{a, r}\right)$ можно естественным образом «поднять» на пространство сечений пучка $\mathfrak{T}_{\mathfrak{g}}$ над произвольным открытым множеством. Таким образом, $\mathfrak{T}_{\mathfrak{g}}$ есть ядерный пучок (некоммутативных) алгебр Фреше с базой $\Delta(\mathfrak{g})$. Разумеется, $\mathfrak{T}_{\mathfrak{g}}=\mathscr{O}$, если $\mathfrak{g}$ есть коммутативная алгебра Ли.

Пусть $X \in \mathscr{U}(\mathfrak{g})-\bmod$ и $\alpha: \mathfrak{g} \rightarrow \mathscr{L}(X)$ - соответствующее лиево представление. Напомним [10,9], что спектр Тейлора $\sigma(\mathfrak{g}, X)$ модуля $X$ определяется как множество тех $\lambda \in \Delta(\mathfrak{g})$, для которых нарушается точность комплекса Кошуля $\mathfrak{g}$-модуля $X$, порожденного представлением $\alpha-\lambda: \mathfrak{g} \rightarrow \mathscr{L}(X)$. Используя предложение 2 , можно сравнить спектры Тейлора и Путинара модуля $X$ относительно пучка $\mathfrak{T}_{\mathfrak{g}}$.

ПРЕДЛОЖЕНИЕ 3. Пусть $X \in \mathscr{F}_{\mathfrak{g}}$-mod. Спектр Тейлора $\mathfrak{g}$-модуля $X$ содержится в спектре Путинара $\mathscr{F}_{\mathfrak{g}}$-модуля $X$ относительно пучка $\mathfrak{T}_{\mathfrak{g}}$, m. е. $\sigma(\mathfrak{g}, X) \subseteq \sigma\left(\mathfrak{T}_{\mathfrak{g}}, X\right)$

Из определения пучка $\mathfrak{T}_{\mathfrak{g}}$ следует, что как пучок пространств Фреше он обладает мягкой резольвентой (1); тем самым, пространство $\Delta(\mathfrak{g})$ превращается в $\mathfrak{T}_{\mathfrak{g}}$-пространство (определение В) и само пространство $\Delta(\mathfrak{g})$ является $\mathfrak{T}_{\mathfrak{g}}$-ацикличным. 
4. Банаховы $\mathscr{F}_{\mathfrak{g}}$-модули. Пусть $A$ - банахова алгебра и $\mathfrak{a}$ - ее конечномерная нильпотентная подалгебра Ли. Мы скажем, что a является сверxнилъпотентной подалгеброй Ли в $A$, если каждый элемент $u \in[\mathfrak{a}, \mathfrak{a}]$ является нильпотентным [11]. Пусть $X$ - банахово пространство и $T=\left(T_{1}, \ldots, T_{m}\right)-$ семейство операторов в $\mathscr{L}(X)$, порождающее конечномерную нильпотентную подалгебру Ли $\mathfrak{g}_{T} \subset \mathscr{L}(X)$. Если $\lambda \in \mathbb{C}^{m}$, то $\mathfrak{g}_{T-\lambda}$ - подалгебра Ли в $\mathscr{L}(X)$, порожденная семейством $T-\lambda$. Спектр Тейлора $\sigma(T)$ операторного семейства $T$ определяется $[10,12]$ как множество тех $\lambda \in \mathbb{C}^{m}$, для которых нарушается точность комплекса Кошуля $\mathfrak{g}_{T-\lambda}$-модуля $X$. Далее, алгебра Ли $\mathfrak{g}_{T}$ является эпиморфным образом некоторой градуированной нильпотентной алгебры Ли $\mathfrak{g}$ с $m$ лиевыми образующими $e_{1}, \ldots, e_{m}$. Пусть $\tau: \mathfrak{g} \rightarrow \mathfrak{g}_{T}, \tau\left(e_{i}\right)=T_{i}$, 一 эпиморфизм алгебр Ли. Тогда $X$ является банаховым $\mathfrak{g}$-модулем относительно $\tau$, т. е. $X \in \mathscr{O}_{\mathfrak{g}}$-mod. При этом, согласно [10], $\sigma(T)=\sigma(\mathfrak{g}, X)$.

Лемма 4. $X \in \mathscr{F}_{\mathfrak{g}}-\bmod$ тогда и толъко тогда, когда $\mathfrak{g}_{T}$ является сверхнильпотентной подалгеброй Ли в $\mathscr{L}(X)$.

Используя предложение 3 и некоторые свойства параметризованных комплексов (см. [2,3] по поводу коммутативного случая), получим следующее предложение.

ПрЕДЛОЖЕНИЕ 5. Пусть $X \in \mathscr{F}_{\mathfrak{g}}-\bmod -$ банахов модуль. Тогда $\sigma(\mathfrak{g}, X)=$ $\sigma\left(\mathfrak{T}_{\mathfrak{g}}, X\right)$.

5. Функциональное исчисление. Сформулируем теперь основной результат заметки - теорему о функциональном исчислении.

Теорема 6. Пусть $X$ - банахово пространство, $T \subset \mathscr{L}(X)$ - конечное семейство операторов, порождающих сверхнилъпотентную подалгебру Ли $\mathfrak{g}_{T}$ в $\mathscr{L}(X)$ и $U$ - открытая окрестность спектра Тейлора $\sigma(T)$ семейства $T$. Тогда $\mathfrak{g}_{T}=\alpha(\mathfrak{g})$ и $T=\alpha(e)$, где $\mathfrak{g}-$ конечномерная нильпотентная алгебра Ли, допускающая положительную градуировку с лиевым семейством образующих е, и $\alpha: \mathfrak{g} \rightarrow \mathscr{L}(X)$ - лиево представление. Более того, существует $\mathfrak{T}_{\mathfrak{g}}(U)$-исчисление для семейства операторов $T$, m. е. имеется непрерывный унитальный гомоморфизм алгебр $\tilde{\alpha}: \mathfrak{T}_{\mathfrak{g}}(U) \rightarrow \mathscr{L}(X)$ из алгебры Фреше $\mathfrak{T}_{\mathfrak{g}}(U)$ всех формально радикальных функций на $U$ от элементов алгебры Ли $\mathfrak{g}$ в банахову алгебру $\mathscr{L}(X)$, продолэсающий представление $\alpha$.

Доказательство следует из теоремы 1, леммы 4 и предложения 5.

В частности, если $T$ - коммутативное семейство $m$ операторов, то коммутативная алгебра Ли $\mathfrak{g}_{T}$ является образом представления $\alpha: \mathbb{C}^{m} \rightarrow \mathscr{L}(X)$, $\alpha\left(z_{i}\right)=T_{i}$, и теорема 6 сводится к тейлоровскому голоморфному исчислению [1].

Автор глубоко признателен Ю. В. Туровскому и А. Ю. Пирковскому за подробное обсуждение результатов и Э. Альбрехту, А. Я. Хелемскому и Й. Эшмаеру за внимание и интерес к данной работе.

\section{ЛитерАТУрА}

1. Taylor J. L. Adv. in Math., 9, 183-252 (1972). 2. Хелемский A. Я. Гомология в банаховых и топологических алгебрах. МГУ, М., 1986. 3. Eschmeier J., Putinar M. Spectral decompositions and analytic sheaves. Clarendon Press, Oxford Univ. Press, 1996. 4. Годеман P. Алгебраическая топология и теория пучков. ИЛ, М., 1961. 5. Хёрмандер Л. Введение в теорию функций нескольких комплексных переменных. Мир, М., 1968. 6. Досиев А. А. Функц. анализ и его прил., 34, вып. 4, $82-84$ (2000). 
7. Dosiev A. A. J. Math. Sciences. 124, вып. 2, 4886-4908 (2004). 8. Хелемский A. Я. Банаховы и полинормированные алгебры: общая теория, представления, гомологии. Наука, М., 1989. 9. Досиев А. А. Функц. анализ и его прил., 37, вып. 1, 73-77 (2003). 10. Fainshtein A. S. J. Operator Theory, 29, 3-27 (1993). 11. Туровский Ю. В. Функц. анализ и его прил., 18, вып. 2, 77-78 (1984). 12. Dosiev A. A. J. Operator Theory, 48, вып. 3, 585-614 (2002).

Department of Mathematics, Atilim University, Ankara e-mail: adosiev@atilim.edu.tr,dosiev@yahoo.com

Поступило в редакцию 10 декабря 2003 г.

УДК 512.622

\title{
Очень гиперболические многочлены
}

\author{
(c) 2005. В. П. Костов
}

Посвящается памяти академика А. А. Болибруха

1. Введение. В этой работе мы рассматриваем вещественные многочлены одной вещественной переменной. Многочлен называется гиперболическим (соответственно строго гиперболическим), если все его корни вещественные (соответственно вещественные и отличны друг от друга). Производная гиперболического многочлена степени $\geqslant 2$ гиперболична. Многочлен $f$ называется первообразной порядка $k$ многочлена $g$, если $f^{(k)}=g$. Гиперболический многочлен $(Г \mathrm{M})$ называется оченъ гиперболическим, если он имеет гиперболические первообразные всех порядков. Вектор кратности (BK) заданного ГМ - это вектор с компонентами, равными кратностям различных корней рассматриваемого многочлена и расположенными в порядке возрастания. Пример: BK многочлена $x^{2}(x-1)$ равняется $(2,1)$.

ПримеР 1. Все ГМ степени $\leqslant 3$ очень гиперболические: ГМ степени 1 (соответственно 2) интегрируется до ГМ степени 2 (соответственно 3 ); если у ГМ третьей степени ВK равен $(1,1,1)$ (соответственно $(2,1),(1,2),(3))$, то, интегрируя всегда от среднего корня (соответственно от корня кратности $>1$ ), можно получить первообразные с $\mathrm{BK}(1, k, 1)$ (соответственно $(k, 1),(1, k),(k))$. ГМ $x^{k}(x-1)^{2}$ для $k \geqslant 2$ не имеет гиперболической первообразной.

Теорема 2. Для любого $n \in \mathbb{N}^{*}$ существуют строго и очень гиперболический многочлен степени $n$ и постоянные интегрирования (ПИ) $c_{i}$, такие, что

(a) первообразные всех порядков с ПИ $c_{i}$ строго и очень гиперболичны;

(b) для $i \in \mathbb{N}^{*}$ существуют окрестности $\Delta_{i}$ чисел $c_{i}$, такие, что первообразные всех порядков с любыми ПИ $d_{j} \in \Delta_{i}$ строго и очень гиперболичны.

Для семейства многочленов $P(x, \tilde{a})=x^{n}+a_{1} x^{n-1}+\cdots+a_{n}, x, a_{i} \in \mathbb{R}$, положим $H_{n}=H_{n}(\tilde{a})=\left\{\tilde{a} \in \mathbb{R}^{n} \mid P\right.$ гиперболичен $\}$ (область гиперболичности) и $D_{n}=D_{n}(\tilde{a})=\left\{\tilde{a} \in \mathbb{R}^{n} \mid P\right.$ очень гиперболичен $\}$. Мы полагаем $a_{1}=0$, чего можно добиться сдвигом переменной $x$, и $a_{2}=-1$ (поскольку растяжения $x \mapsto e^{t} x, a_{j} \mapsto e^{j t} a_{j}, t \in \mathbb{R}$, сохраняют области $H_{n}$ и $D_{n}$, а $H_{n} \cap\left\{a_{2}>0\right\}=\varnothing$, $\left.H_{n} \cap\left\{a_{2}=0\right\}=D_{n} \cap\left\{a_{2}=0\right\}=0\right)$. 